\title{
Influence of Aspect Ratio on Thermal Performance of Heat Sink using Ansys
}

\author{
P. C. Mukesh Kumar'iand C. M. Arun $\operatorname{kumar}^{2 \dagger}$ \\ ${ }^{1}$ Mechanical Department, University College of Engineering, Dindigul, Tamilnadu, 624622, India \\ ${ }^{2}$ ECE Department, University College of Engineering, Pattukottai, Tamilnadu, 614701, India \\ †Corresponding Author Email: cmarunkumar@gmail.com
}

(Received May 15, 2018; accepted July 18, 2018)

\begin{abstract}
The integrated circuits face a huge issue to meet out the cooling demand due to the rapid development in technology. Several researchers have investigated the different possibility of cooling medium to improve the heat dissipation in an integrated circuits. Heat sink is a kind of thermal heat transfer device used to dissipate heat from an integrated circuit (IC) to surrounding due to low cost and reliability in heat dissipation. In this numerically work, the electronic chip with the heat sink is analyzed to study about the cooling rate, surface temperature of the chip, reliability and power dissipation of the chip with different heat transfer medium, fin height and fin thickness. The different heat transfer medium is air, water and engine oil. The ANSYS (v12) fluent software is used to study numerically about the electronic chip cooling. In this research work, the heat transfer rate of water is $9.5 \%$ greater than air and $1.4 \%$ than engine oil at the same Reynolds number is obtained. The power dissipation is increases up to $1.45 \%$ of the fin height $55 \mathrm{~mm}$ and heat transfer rate is enhanced by increasing the fin thickness up to $2.10 \%$ in $6 \mathrm{~mm}$. However, the lifetime of the electronic chip with fin height $55 \mathrm{~mm}$ is $2.06 \% \mathrm{hrs}$ (day) greater than the fin with $35 \mathrm{~mm}$. It is observed that the electronic chip with water as a heat transfer medium with proper fin height and thickness is highly reliable to enhance the heat transfer than that of air and engine oil.
\end{abstract}

Keywords: ANSYS-Fluent; Heat sink; Nusselt number and Fin height.

\section{NOMENCLATURE}

$\begin{array}{ll}\mathrm{A} & \text { area }\left(\mathrm{m}^{2}\right) \\ \mathrm{C}_{\mathrm{p}} & \text { specific heat capacity }\left(\mathrm{Jkg}^{-1} \mathrm{k}^{-1}\right) \\ \mathrm{h} & \text { convection coefficient }\left(\mathrm{Wm}^{-2} \mathrm{k}^{-1}\right) \\ \mathrm{k} & \text { thermal conductivity of the heat sink } \\ \mathrm{L} & \begin{array}{l}\text { material }\left(\mathrm{Wm}^{-1} \mathrm{k}^{-1}\right) \\ \text { characteristic length }(\mathrm{m})\end{array} \\ \mathrm{L} & \text { fin length } \\ \mathrm{N} & \text { number of fins } \\ \mathrm{Nu} & \text { Nusselt number } \\ \mathrm{Pr} & \text { Prandtl number } \\ \mathrm{Q} & \text { total heat flow }(\mathrm{W}) \\ q^{r r} & \text { heat transfer flux }\left(\mathrm{Wm} \mathrm{W}^{-2}\right) \\ \mathrm{Re} & \text { Reynolds number } \\ \mathrm{R} & \text { thermal resistance }\left({ }^{\circ} \mathrm{C} / \mathrm{W}\right) \\ \mathrm{T} & \text { temperature }\left({ }^{\circ} \mathrm{C}\right) \\ \mathrm{T}_{\mathrm{b}} & \text { surface temperature of the electronic chip } \\ \mathrm{T}_{\mathrm{o}} & \text { environmental air temperature }\end{array}$

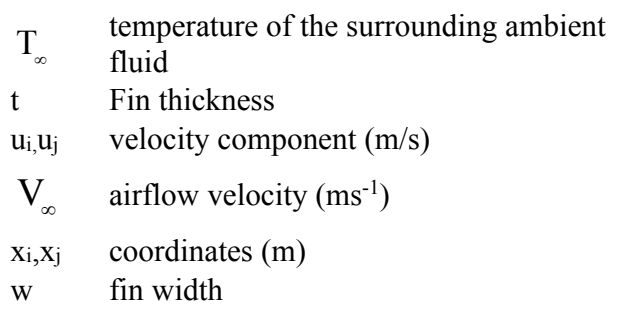

\section{Greek letters}

$\varepsilon \quad$ total, hemispherical emissivity

$\mu \quad$ dynamic viscosity $\left(\mathrm{kg} \mathrm{m}-{ }^{1} \mathrm{~s}^{1}\right)$

$v \quad$ kinematic viscosity $\left(\mathrm{m}^{2} / \mathrm{s}\right)$

$\rho \quad$ density $\left(\mathrm{kg} / \mathrm{m}^{3}\right)$

$\sigma \quad$ Stefan-Boltzmann constant $\left(\sigma=5.670 \times 10^{-8} \mathrm{~W} / \mathrm{m}^{2} . \mathrm{K}^{4}\right)$ 


\section{INTRODUCTION}

The rapid developments in the design of compact electronic circuits have resulted in increase in a large amount of heat generated per unit volume. In addition, as the size of the components decreases then there is a dramatical increase in heat flux per unit areas. The heat sink is played an important role to dissipate heat from a high-temperature heat source to a lower temperature surrounding. It is notable that the performance of the chip, reliability, power consumption and lifetime are reciprocally proportional to the internal temperature. Among various types of heat sink $\mathrm{s}$, the aluminum heat sink $s$ provide more reliable and cheapest one to achieving a large total heat transfer surface area without excessive primary surface area. Tuckerman and Pease (1981) revealed that cooling capacities for liquid cooled $1 \times 1 \mathrm{~cm}^{2}$ end-to-end microchannel heat sinks of up to $790 \mathrm{~W} / \mathrm{cm}^{2}$ with an internal thermal resistance of $0.09 \mathrm{~cm}^{2} \mathrm{~K} / \mathrm{W}$ for a flow rate of $0.6 \mathrm{l} / \mathrm{min}$ and a pressure drop of two bars. Raghuraman et al. (2017) studied numerically about the enhancement of heat and fluid flow characteristics in rectangular microchannel heat sinks (MCHS) based on various aspect ratios. The working fluid considered for the analysis is water. The fluid flow and heat transfer characteristics of a three-dimensional MCHS are obtained numerically by solving the appropriate governing equations. Rathanasamy and Kalaivanan (2010) conducted experiments related to forced convection through rectangular microchannels. Wong and Ghazali (2012) simulated a CFD model of a microchannel heat sink to study fluid flow and heat transfer phenomena across the MCHS. The channels in their analytical work were rectangular and made from silicon. They analyzed the model for flows up to Reynolds number of 400 and obtained temperature and heat flux distribution along the channel length.

Naphon and Khonseur (2009) studied about the pressure drop and heat transfer characteristics along a microchannel heat sink for constant heat flux operating condition. They examined the heat transfer characteristics by varying the length and width of the microchannel and reported that the channel dimension has played a crucial role in the heat transfer characteristics and performance of the micro-channel heat sink. Raghuraman et al. (2013) carried out experiments on rectangular high aspect ratio copper microchannel heat sink using $\mathrm{TiO}_{2}$ nano fluid as the working fluid and they investigated the thermal and fluid flow characteristics across the MCHS. Dharaiya and Kandlikar (2012) developed correlations for the Nusselt number for heat transfer in rectangular microchannels for both developing and fully developed laminar flows and this technique was very much useful for the design and optimization of microchannel heat sinks and other microfluidic devices.

Currently, liquid electronic cooling methods are being focused with the different heat transfer liquid medium. It proposed by nanofluids is being investigated in electronic cooling systems.
Muruganandam and Mukesh Kumar (2017), Palanisamy and Mukesh Kumar (2017) and Vijayakumar and Mukesh Kumar (2017) experimental analysis the effect of $\mathrm{Al}_{2} \mathrm{O}_{3}$ nano fluids in the heat exchanger and reported that being $\mathrm{Al}_{2} \mathrm{O}_{3}$ nano fluids are better than the alternative liquid coolant water.

Xie et al. (2009) carried out a numerical study in the mini-channel heat sinks to study the heat transfer characteristics of laminar flow. They optimized the geometry using water as the working fluid for effective heat dissipation and lesser pressure drop. Liu and Garimella (2004) conducted an experimental and numerical study on the flow field and pressure drop for water in rectangular microchannel. Their results show that the conventional correlations provide reliable predictions for the laminar flow.

Li and Byon (2015) experimentally and numerically studied on the orientation effect for a radial heat sink with a circular base, concentric ring, and rectangular fins. They investigated the effects of orientation angle with respect to gravity, various geometric parameters (fin number, fin length, fin height, and height of the base), Elenbaas number, on the Nusselt number. Their results showed that the influences of fin number and fins length on the orientation effect are significant, whereas the fin height and base height are slightly insignificant. Srinivasan (2017) studied and analysed about the missile with grid fins and the effect on flow drag using ANSYS. Godwin (2017) and Lakshmanan (2017) investigated about the optimum parameters for obtaining the best performance using alternate fuels of IC engines working under the current cooling system using Nanofluids.

It is clear from the literature survey that, little work has been done on the cooler heat sink in the sense of water, and air heat dissipation analysis based on aspect ratios. In this study, heat transfer of a heat sink used in the electronic chip is investigated numerically and variable convective heat transfer coefficient with the different aspect ratio is studied. The effect of internal heat generation and radiation from heat sink with respect to the change of thickness and height is investigated. Therefore, in this research work thermal performance of the cooler heat sink is carried out by using ANSYSFluent Software and replacing the conventional heat sink to meet out the objectives: a) to investigate numerical studies on Nusselt and Reynolds number. b) To study the heat dissipation capacity of the cooler heat sink with respect to aspect ratios of fins. c) To analyze and calculate the reliability and power consumption of electronic chip based on the cooler heat sink.

\section{DESIGN CONCEPT OF THE 3D HEAT SINK MODEL}

Fig.1 shows the schematic representation of the cooler heat sink studied in the present work. The cooler heat sink with the length $49 \mathrm{~mm}$ and width of $45 \mathrm{~mm}$ and a total height of $29 \mathrm{~mm}$ is simulated 
along with one inlet and outlet. In this work, the thermal performances of cooler heat sinks with cooler sections using air, water, and engine oil as heat transfer fluid are studied numerically to investigate the effects based on different heat transfer boundary conditions. Base plate thickness, fins thickness, length, and width is accounts for the uniform distribution of heat through the base of the heat sink since electronic chips are littler than the heat sinks.

Kandasamy et al. (2008) investigated a phase change material (PCM)-based heat sink in the transient thermal management of plastic quad flat package (QFP) electronic devices experimentally and numerically. Their Results showed that increased power inputs enhance the melting rate as well as the thermal performance of the PCM-based heat sink $\mathrm{s}$ until the PCM is fully melted. The heat transfer mechanism of the heat sink is to conduct heat from the top surface of chip to the base of sink by its thermal conduction and then dissipate heat to the air by the effect of thermal convection or convectionradiation. Lober (1999) reported the use of CFD thermal modelling software in thermal management which optimally integrated the design and reduced the design cycle time. Chang et al. (2000) studied the $30 \mathrm{~W}$ socket CPU of a desktop computer to cool with minimum air flow rate and heat sink size. Sivakumar et al. (2017) observed that the ventilation problems in rooms with different shapes of inlets, outlets and heights can be solved effectively by using numerical calculations and CFD software.

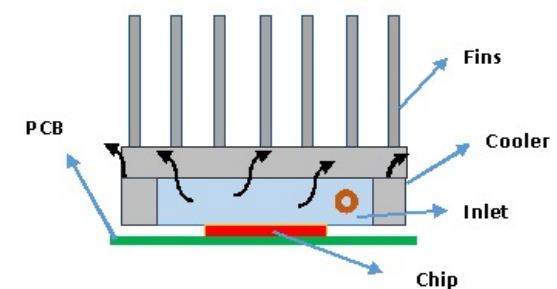

Fig. 1. Schematic diagram electronic chip with heat sink $s$

In this study, the base heat sink has seven fins with a $20 \mathrm{~mm}$ height. In general, the heat sink is made from either aluminum or copper but also made from other metals such as silver and steel. The thermal conductivity of each material is different from each other, based on this the heat conduction performance also varies from high to low, silver, copper, aluminum, and steel. In addition to that, the usage of silver and copper as heat sink would be too expensive and thermal conductivity of steel is very low. The heat sinks has a fin height of $20 \mathrm{~mm}$, $35 \mathrm{~mm}, 40 \mathrm{~mm}, 45 \mathrm{~mm}, 50 \mathrm{~mm}$, and $55 \mathrm{~mm}$. The fin thickness is $2 \mathrm{~mm}, 5 \mathrm{~mm}, 7 \mathrm{~mm}, 8 \mathrm{~mm}$ and $10 \mathrm{~mm}$ respectively and spacing was kept constant at $3 \mathrm{~mm}$. Fig. 2 and fig 3 show the schematic cooler heat sink with $20 \mathrm{~mm}$ and $50 \mathrm{~mm}$ fins. The rectangular cooler box with an inlet and outlet is fixed in the base of the heat sink have dimensions of $39 \mathrm{~mm} x$ $45 \mathrm{~mm}$. This rectangular cooler box is used to transfer the heat from the top of the chip to the surrounding through convection methods.

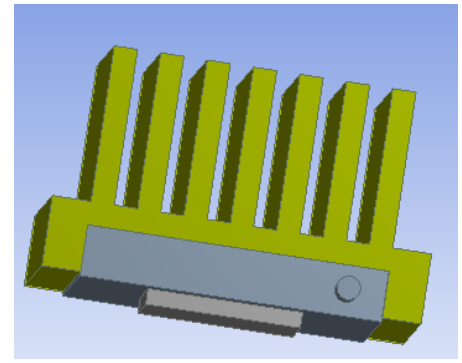

Fig. 2. Schematic diagram of heat sink with $20 \mathrm{~mm}$ fin height

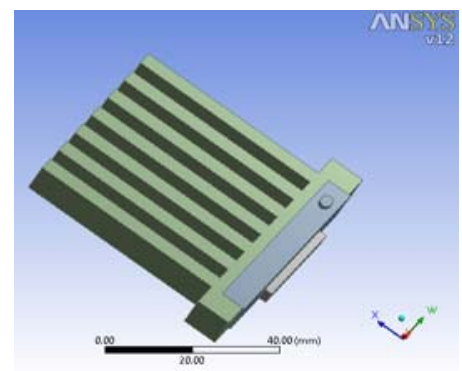

Fig. 3. Schematic diagram of heat sink with $50 \mathrm{~mm}$ fin height.

The Mesh analysis of cooler heat sink with $20 \mathrm{~mm}$ and $50 \mathrm{~mm}$ is shown in fig 4and 5.Here, the mesh analysis is carried out to obtain a suitable mesh size and grid, to provide an accuracy output with respect to computational time. After the determination of mesh, the boundary conditions are applied in the model design to obtain the accurate results.

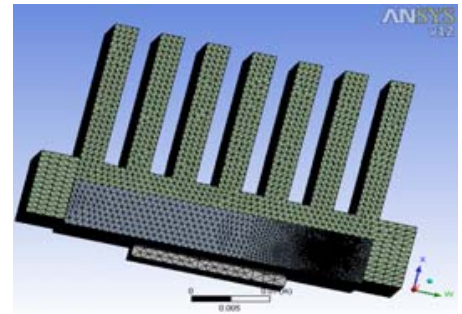

Fig. 4. Mesh structure of cooler heat sink for $20 \mathrm{~mm}$.

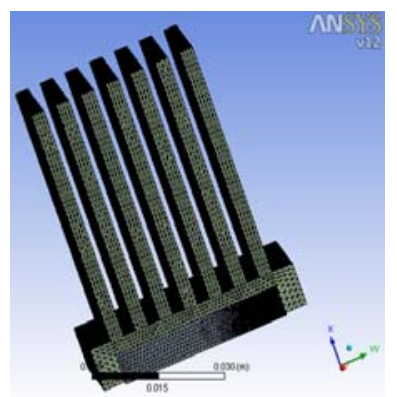

Fig. 5. Mesh structure of cooler heat sink for $50 \mathrm{~mm}$.

\subsection{Governing equations}

The Governing continuity, momentum and energy equations for the fluid can be written as:

$\frac{\partial u}{\partial x}+\frac{\partial v}{\partial y}+\frac{\partial w}{\partial z}=0$ 


$$
\rho u_{j} \frac{\partial u_{i}}{\partial x_{j}}=-\frac{\partial p}{\partial x_{i}}+\frac{\partial}{\partial x_{j}}\left[\mu_{t}\left(\frac{\partial u_{i}}{\partial x_{j}}+\frac{\partial u_{j}}{\partial x_{i}}\right)\right]
$$

Energy equations

$\rho u_{j} \frac{\partial \tau}{\partial x_{j}}=\frac{\partial}{\partial x_{j}}\left[\frac{\partial \tau}{\partial x_{j}}\left(\frac{\mu_{2}}{\sigma_{2}}+\frac{\mu_{\tau}}{\sigma_{\tau}}\right)\right]$

The heat flow is mathematically givens as

$Q=-k A d T / d x$

Where, Q-W(Watt), A-m ${ }^{2}, \mathrm{~d}$ T- ${ }^{\circ} \mathrm{C}$ or K

The flow is due to the buoyant forces caused by the change of temperature in the fluid body, then the methods are known as free or natural convection.

Heat flow,

$$
Q=h A\left(T_{1}-T_{2}\right)=\frac{T_{1}-T_{2}}{1 / h A}
$$

The term $1 / \mathrm{hA}$ is called convection resistance to heat flow. The heat transfer coefficient $h$ is calculated by

$$
h=\frac{Q}{A\left(T_{1}-T_{2}\right)}
$$

The fin tip can be assumed to be adiabatic, and the condition at the fin tip can be expressed as

$$
\frac{d \theta}{d x}=0 \text { at } x=L
$$

The rate of heat transfer from the fin can be determined from Fourier's law of heat conduction

$$
Q_{\text {adiabatictip }}=\sqrt{h p k A}\left(T_{b}-T_{\infty}\right) \tanh m L
$$

Convection from fin tip is

$$
\begin{aligned}
& Q_{\text {adiabatic tip }}= \\
& \sqrt{h p k A}\left(T_{b}-T_{\infty}\right) \frac{\sinh m L+(h / m k) \cosh m L}{\cosh m L+\left(\frac{h}{m k}\right) \sinh m L}
\end{aligned}
$$

$Q=$

$$
N(k A c n \Delta t) \frac{\sinh m L+(h / m k) \cosh m L}{\cosh m L+\left(\frac{h}{m k}\right) \sinh m L}
$$

From the equation 10, the heat flow rate from the fins tip is calculated.

The fin with finite insulated tip is calculated using

$$
Q=N(k A c n \Delta t) \tanh (n L)
$$

\section{$2.2 \quad$ Boundary Conditions}

The boundary conditions are given as inputs for the model in ANSYS - CFD Simulation. The flow of fluid is based on laminar and the corresponding boundary conditions are given below. The coolant is used in this numerical calculation through the inlet is air, water, and engine oil and its corresponding thermo-physical properties are obtained at various temperature.

1. The water or air or engine oil enters the heat sink with constant and uniform velocity.

2. The initial temperature is $26.8^{\circ} \mathrm{C}$.

3. A uniform heat flux is assigned to the bottom wall surfaces from the chip.

4. In the model, the no-slip boundary condition is assigned to all the surfaces.

Rasouli et al. (2015) suggested that the heat transfer and pressure drop of single-phase liquid flow is characterized in eight micro pin fin heat sinks with varied pitch and aspect ratios. The result shows that correlation for the unsteady regime significantly decreased dependency of Nusselt on the Prandtl number compared to the non-vortex-shedding condition.

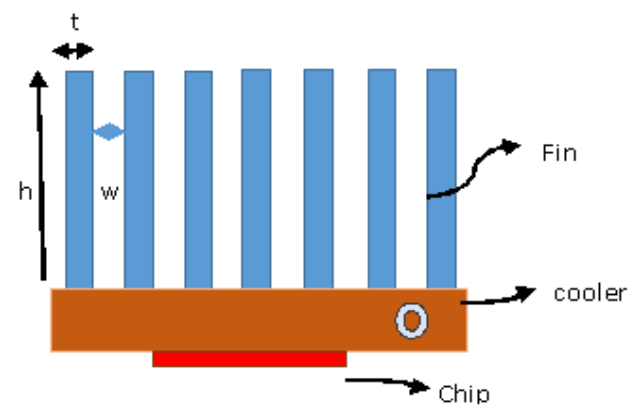

Fig. 6a. Heat sink fin with convection at the tip and geometric representations.

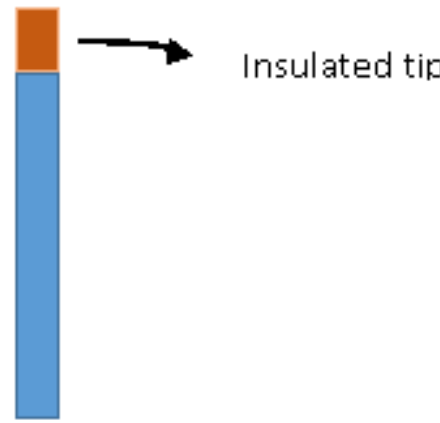

Fig. 6b. Fin with Insulated tip

\section{RESULTS AND DISCUSSION}

Numerical studies were carried out for different aspect ratios corresponding to the length, thickness, and width of the fins. In this numerical validation study, the geometric parameters such as length, width, and thickness are shown in fig.6a.Fig $6 \mathrm{~b}$ shows that the fin with insulated at the end of the tip. In case of the forced convective heat sink, the thermal performance of heat sink is known to non-linearly change as the fin height increases, because the fin efficiency significantly changes as the fin height changes. However, in case of natural convective heat sinks, the fin efficiency is nearly unity, regardless of the fin height due to the low 
heat transfer coefficient (2016).According to this the thermal performance of natural convective heat sink almost linearly increases as the fin height increases.

In general, the heat sink is usually made up of either copper or aluminum. These materials allow heat to pass through quickly and are used because they have many desirable properties for thermally efficient and durable heat exchangers. The heat dissipation is more based on the change of length and thickness as shown in fig 7. This indicates that the heat dissipation rapidly decreased as the fin thickness increased. The fin height has a little influence on the temperature of the cooler heatsink. The thermal performance of the heat sink linearly increased as the fin height increases.

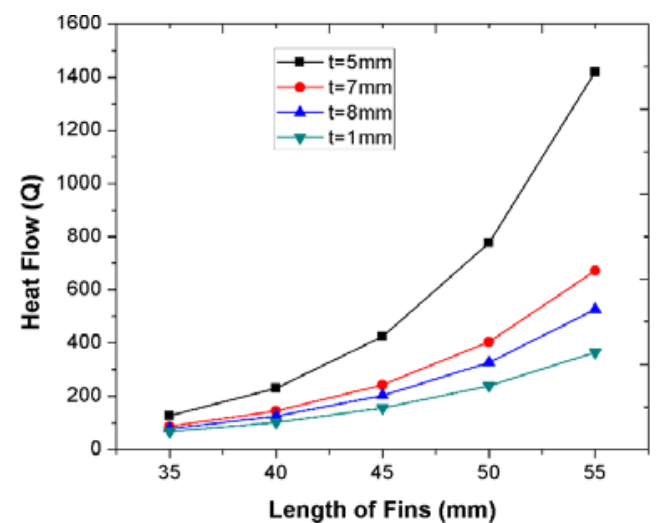

Fig. 7. Effect of heat flow based on an increase of fins length with a change of thickness.

Fig $8 \mathrm{C}$. shows the rate of heat transfer from the fin decreased with increasing of fin thickness.Fin subjected to convection with and without insulated tips is shown in fig 8(a). As shown in fig 8(b), the heat transfer has an improvement with insulated tips compare to the actual fins with convection at the tips. Waleed Al-Sallami et al. (2016) studies show that perforating the strip fins provides an effective and practical means of enhancing heat transfer yet further with additional, significant benefits in terms of reduced pressure losses and heat sink mass and materials consumption.

Mohan and Govindarajan (2011) investigated about the number of fins and their distribution, fin material and base plate thickness for enhancing the heat dissipation rate from CPU. They concluded the results with the help of CFD simulations. Due to the limited space of a heat sink in a PCB Board, it is not possible to increase too much height of the heat sink. So, the base plate is included in heat sink to enhance the heat dissipation rather than increasing the height of heat sink.

The performance of the heat sink is improved by increasing the thickness of fins in an alternative of increasing the number of fins as pointed by Mohan and Govindarajan (2011).

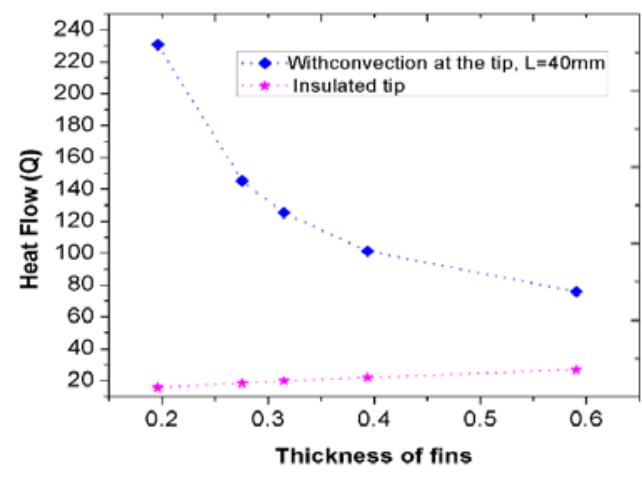

Fig. 8. (a) Fins convection and insulated at the end with length $40 \mathrm{~mm}$.

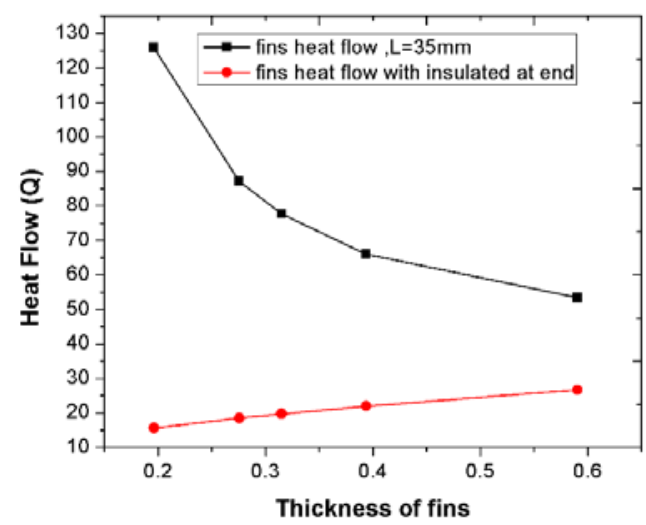

Fig. 8. (b) Fins convection and insulated at end with length $35 \mathrm{~mm}$

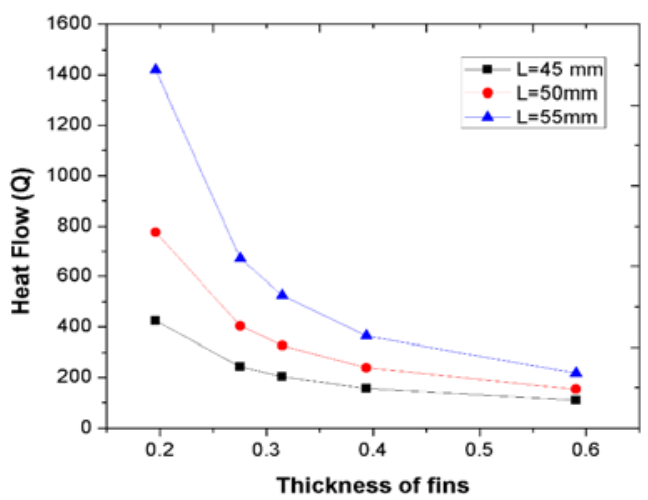

Fig. 8. (C) Influence of heat flow rate based on the aspect ratio of length and thickness of fin with constant width.

It's seen from fig 10 that the thermal resistance of the heat sink decreases with increase in fin length due to the enlarged extended surface area. In addition to that, the thermal resistance is comparatively larger compared to the less thickness of fins. Al-Damook et al. (2015) reported that the use of multiple pin perforations can have substantial performance benefits by enabling the heat transfer to be increased while at the same time reducing both the pressure drop across the heat sink and fan power needed to pump the air through them.

It can be seen in fig 11, the Nusselt number increase when the Reynolds number increases and the corresponding flow of velocity of fluids are also 
calculated. The figure shows that the Nusselt number of water reaches a maximum value of 591.8 when the Reynolds number is at maximum value. The total heat dissipation in heatsink by air is $2 \%$ lesser than other two types of fluids, because of lesser Nusselt number which indicates poorer convective heat transfer.

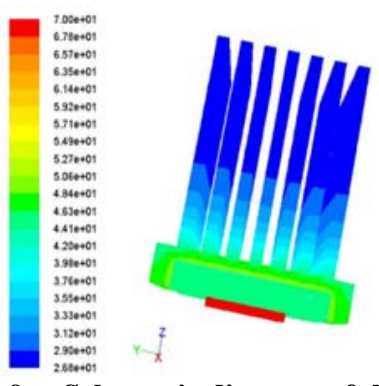

NWSYS

Fig. 9 a. Schematic diagram of the heatsink with $50 \mathrm{~mm}$ height fins

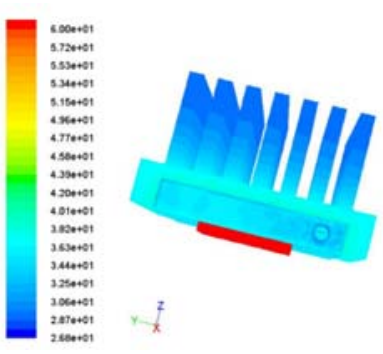

Fig. 9 b. Schematic diagram of heatsink with $20 \mathrm{~mm}$ height fins.

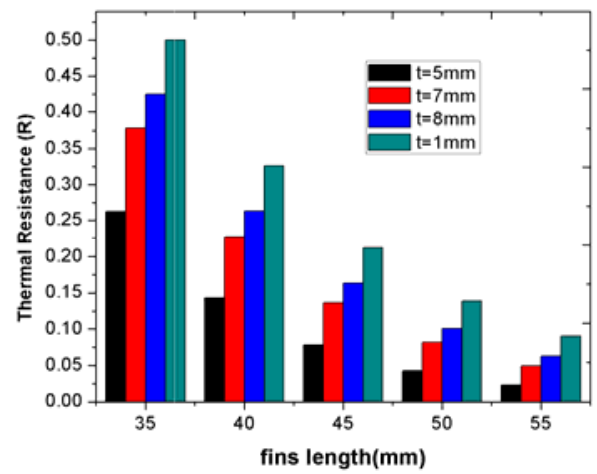

Fig. 10. Thermal resistances of fins with respect to thickness.



Fig. 11. Variation of the Nusselt number at the heat sink with Reynolds number.

Numerical results show that the variations of heat dissipation in the heat sink are depended on the flow velocity of air, water, engine oil from $0.2 \mathrm{~m} / \mathrm{s}$ to $1.5 \mathrm{~m} / \mathrm{s}$ as shown in fig 12 . The heat generation in the electronic chip is simulated by the uniform heat flux at the bottom wall.

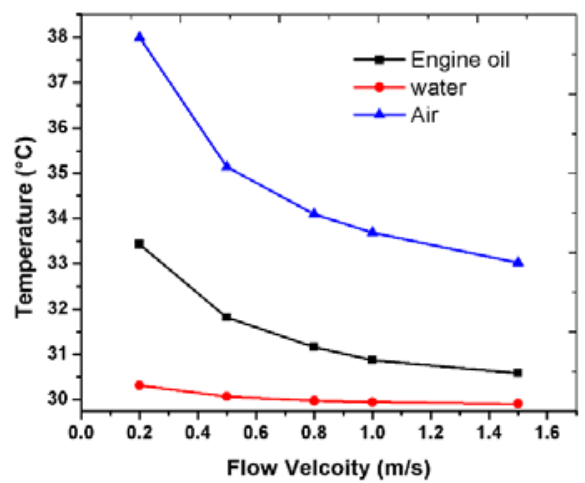

Fig. 12. Variations of chip surface temperature based on the different flow velocity of air, water, and engine oil.

The high flow velocity was able to improve the heat transfer performance of the cooler heatsink. This shows that the nusselt number increases with an increase of flow velocity and heat transfer rate of the heat sink also gradually increase.

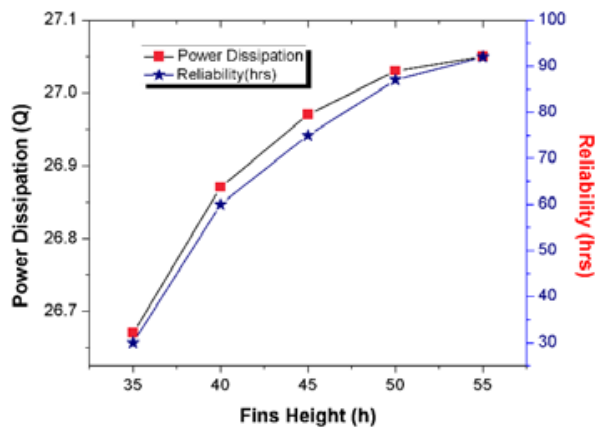

Fig. 13. Power consumption based on fin length.

It's found from Fig. 13 that the numerical studied shows that the power dissipation and reliability of electronic chip is more when the fin height is increased. It is observed that the performance of the heatsink in an electronic chip is increased by increasing the thickness and height of fins instead of increasing the number of fins because of better air flow path between the fins.

\section{CONCLUSION}

In this research work, the cooling performance of an electronic chip is studied with different fin thickness and length by using ANSYS -Fluent (v12).The cooling rate, chip surface temperature, power dissipation and reliability of chip are compared with air, water, and engine oil as heat transfer medium. It is observed that the heat transfer rate of water is $9.5 \%$ greater than air and $1.4 \%$ than engine oil at the same Reynolds number. The power dissipation increases up to $1.45 \%$ of the fin height $55 \mathrm{~mm}$ and heat transfer rate is enhanced by increasing the fin thickness up to $2.10 \%$ in $6 \mathrm{~mm}$. However, the lifetime of the electronic chip with fin height $55 \mathrm{~mm}$ is $2.06 \%$ hrs (day) greater than the fin 
with $35 \mathrm{~mm}$. It is observed and concluded that the electronic chip with water as a heat transfer medium with proper fin height and thickness is highly reliable to enhance the heat transfer than that of air and engine oil.

\section{REFERENCES}

Al-Damook, A., N. Kapur, J. Summers, and H. Thompson (2015). An experimental and computational investigation of thermal air flows through perforated pin heat sinks. Applied Thermal Engineering 89, 365-376.

Chang, J. Y. (2000). Identification of minimum air flow design for a Desktop computer using CFD modelling. $7^{\text {th }}$ intersociety conference on thermal and thermomechanical Phenomena in Electronic systems 1, 330-338.

Dharaiya, V. V. and S. G. Kandlikar (2012). Numerical investigation of heat transfer in rectangular microchannels under $\mathrm{H}_{2}$ boundary condition during developing and fully developed laminar flow. Journal of Heat Transfer 134 (2), 020911

Godwin Antony, A., S. Dinesh, K. Rajaguru, and V. Vijayan (2017). Analysis and Optimization of Performance Parameters in Computerized I.C. Engine Using Diesel Blended with Linseed oil and Leishmaan's Solution. Mechanics and Mechanical Engineering 21(2), 193-205.

Kandasamy, R., X. Q. Wang, and A. S. Mujumdar (2008). Transient cooling of electronics using phase change material (PCM)-based heat sinks. Applied Thermal Engineering 28 (8-9), 10471057.

Lakshmanan, P., P. Kaliyappan, M. Ranjithkumar, K. Aravinth, D. Vakkachan, C. Moorthy, and S. Kumar (2017). An Experimental Investigation to Study the Performance and Emission Characteristics of Chicken Fat Oil Fuelled DI Diesel Engine, Journal of Applied Fluid Mechanics 10, 85-91.

Li, B. and C. Byon (2015). Experimental and numerical study on the heat sink with radial fins and a concentric ring subject to natural convection. Applied Thermal Engineering 90, 345-351.

Liu, D. and S. V. Garimella (2004). Investigation of liquid flow in microchannels, Journal of Thermophysics and Heat Transfer 18, 65-72.

Lober, D. (1999). Optimizing the integration of an electronics system into an existing enclosure using CFD modeling techniques. International Journal of microcircuits and electronic packaging 22, 146-151.

Mohan, R. and P. Govindarajan (2011). Experimental and CFD analysis of heat sinks with base plate for CPU cooling. Journal of Mechanical Science and Technology 25 (8), 2003-2012.

Muruganandam, M. and P. C. Mukesh Kumar (2017). Experimental Analysis of Four Stroke Diesel Engine by using Carbon Nano Tubes Based Nano Fluids as a Coolant. Journal of Applied Fluid Mechanics 10, 1-5.

Naphon, P. and O. Khonseur (2009). Study on the convective heat transfer and pressure drop in the micro-channel heat sink. International Communications in Heat and Mass Transfers 36 (1), 39-44.

Palanisamy, K. and P. C. Mukesh Kumar (2017). Heat Transfer Enhancement and Pressure Drop Analysis of a Cone Helical Coiled Tube Heat Exchanger using MWCNT/Water Nanofluid. Journal of Applied Fluid Mechanics, 10, 7-13.

Raghuraman, D. R. S., R. Thundil Karuppa Raj, P. K. Nagarajan, and B.V.A. Rao (2017). Influence of aspect ratio on the thermal performance of rectangular shaped micro channel heat sink using CFD code. Alexandria Engineering Journal 56, 43-54.

Raghuraman, D. R. S., P.K. Nagarajan, and S. Suresh (2013). Thermal performance of higher aspect ratio microchannels using $\mathrm{TiO}_{2}$ water nanofluids. Journal of Nanoscience and Nanotechnology 13 (4), 2842-2846.

Rathanasamy, R., and K. Kalaivanan (2010). Experimental investigation of forced convective heat transfer in rectangular microchannels. ARPN Journal of Engineering and Applied Sciences 5 (5), 21-26.

Rasouli, E., C. Naderi, and Vinod Narayanan (2018). Pitch and aspect ratio effects on singlephase heat transfer through microscale pin fin heat sinks. International Journal of Heat and Mass Transfer 118, 416-428.

Sivakumar, V., K. Visagavel, and A. Selvakumar (2017). Analysis of Ventilation Rate in Cross Ventilated Rooms by Varying Aperture Shape of Windows using CFD. Journal of Applied Fluid Mechanics 10, 61-68.

Srinivasan, R., V. Vijayan, and K. Sridhar (2017). Computational Fluid Dynamic Analysis of Missile with Grid Fins. Journal of Applied Fluid Mechanics. 10, 33-39.

Tuckerman, D. B. and R. F. W. Pease (1981). Highperformance heat sinking for VLSI. Electron Device Letters 2 (5), 126-129.

Vijayakumar, M. and P. C. Mukesh Kumar (2017). Performance Enhancement and Emissions Analysis of Diesel Engine with Biodiesel, NPropanol and 1-Butanol Blend. Journal of 
P. C. Mukesh Kumar and C. M. Arun kumar/JAFM, Vol. 11, Special Issue, pp. 45-52, 2018.

Applied Fluid Mechanics 10, 79-84.

Waleed, Al-Sallami, AmerAl-Damook, and H.M. Thompson (2016). A numerical investigation of thermal airflows over strip fin heatsink. International Communications in Heat and Mass Transfer 75, 183-191.

Wong, W. H. and N.M. Ghazali (2012). Numerical simulation of a microchannel for microelectronic cooling. Jurnal Teknologi 46 (1), 1-6.

Xie, X. L, Z. J. Liu, Y. L. He, and W. Q. Tao (2009). Numerical study of laminar heat transfer and pressure drop characteristics in a watercooled mini channel heat sink. Applied Thermal Engineering 29(1), 64-74. 